\title{
IOT Medical Device-Assisted Foam Dressing in the Prevention of Pressure Sore during Operation
}

\author{
Yan Meng, ${ }^{1}$ Hui Zhao, ${ }^{2}$ Zhigai Yin, ${ }^{3}$ and Xiaona Qi ${ }^{4}{ }^{4}$ \\ ${ }^{1}$ Operating Room, Xingtai People's Hospital, Xingtai 054001, Hebei, China \\ ${ }^{2}$ Nursing Department, Xingtai People's Hospital, Xingtai 054001, Hebei, China \\ ${ }^{3}$ The Brotherhood of Surgical, Xingtai People's Hospital, Xingtai 054001, Hebei, China \\ ${ }^{4}$ The Emergency Department, Xingtai People's Hospital, Xingtai 054001, Hebei, China \\ Correspondence should be addressed to Xiaona Qi; qixiaona@stu.cpu.edu.cn
}

Received 1 February 2021; Revised 23 February 2021; Accepted 10 March 2021; Published 12 April 2021

Academic Editor: Sang-Bing Tsai

Copyright (C) 2021 Yan Meng et al. This is an open access article distributed under the Creative Commons Attribution License, which permits unrestricted use, distribution, and reproduction in any medium, provided the original work is properly cited.

\begin{abstract}
With the development of the times, people's living standards are constantly improving, but the medical pressure brought by the aging population is also increasing, and pressure ulcers in elderly patients during hospitalization are also constantly occurring. Of course, the relevant medical equipment is also progressing, especially the emergence of medical-assistant foam dressing on the Internet of Things, which makes people cope with pressure ulcers more handily. In order to test the role of IOT medical devices, especially the application of foam dressings in the prevention of pressure ulcers, this article has carried out a survey of patients in a city hospital, investigated relevant literature, interviewed professionals, collected relevant information, designed experiments, and obtained relevant research data. Research shows that IOT medical devices can improve the efficiency of hospital treatment and improve the efficiency by about $12 \%$; pressure ulcer incidence in different age groups of people is different, and the probability of occurrence in the elderly population is far higher than that of young people; foam dressing has obvious effect on the prevention of pressure sore, and the probability of pressure sore after using foam dressing is lower than that of unused $35 \%$. It indicates that foam dressing can play a key role in the prevention of pressure sore.
\end{abstract}

\section{Introduction}

With the continuous development of China's population aging and chronic diseases [1], its harm is increasingly reflected, and the elderly people with chronic diseases bear the brunt. And, the miniaturization of the family structure and the tendency of two generations' separation make the empty-nest elderly more and more prominent. The elderly with heart disease and cerebrovascular disease often die because they cannot be found and rescued in time when they are alone. There are many problems in the daily monitoring and management of chronic diseases in the elderly. At the same time, under the promotion of information and Internet of Things technology, "intelligent" medical products on the market are showing a blowout trend $[2,3]$. The scope of community education services and home care is more and more extensive, and the requirements are also higher and higher. At this stage, the knowledge of pressure ulcer of community and family nursing staff is lacking or updated slowly [4], which directly affects the prevention and treatment of pressure ulcer. Medical Internet of Things can improve the accuracy of data and prolong the life of the network. Because the data in the medical Internet of Things has the characteristics of sudden and large amount of data [5], the event-driven mode is very suitable for the monitoring of emergencies. At the same time, it can also make the nodes not in the monitoring area in the dormant state and reduce the energy consumption of the nodes in the network [6].

According to statistics, the incidence of pressure ulcer in hospitalized patients in China is $3 \% \sim 12 \%$, and the population range is mainly elderly patients, the incidence rate is $10 \% \sim 25 \%$, and the total mortality rate increases 6 times. The incidence rate did not decrease compared with the previous 
one. The occurrence of pressure ulcers aggravates the physiological and psychological pain of patients, causes huge economic losses, and increases the workload of nurses, which leads to some medical disputes and takes up a large number of public health resources. The prevention of pressure ulcer is one of the important problems to be solved urgently in the clinical nursing work and is a very important reference index to evaluate the quality of nursing. There is a global consensus that the prevention of pressure ulcers is the best treatment [7]. The incidence of pressure ulcer in extracorporeal circulation surgery is as high as $16.7 \%$, which has been paid close attention by medical workers. Therefore, experts at home and abroad have certain research on pressure sore [8].

Wang Ying introduced the causes and symptoms of pressure ulcers, elaborated the harm of pressure ulcers to patients, increased the family burden of patients, and explained the reasons why pressure ulcers could not be completely prevented. Based on reducing the probability of pressure ulcers and reducing the harm degree of pressure ulcers, the prevention measures of pressure ulcers were introduced, so as to analyze the probability of pressure ulcers after improvement, which is the phase of pressure ulcers to provide reference for the work [9]. Xu Ting took 10,000 patients as experimental subjects and set them as the experimental group and the control group, respectively. He observed the causes and differences of pressure ulcers in the hospital between the two groups of patients and carried out pressure ulcer prevention for the two groups of patients. According to medical guidelines, it was observed whether the bed sore situation has changed after the implementation of relevant preventive measures [10]. In order to investigate the cause of pressure ulcers, Hui constructed a research model for the prevention of pressure ulcers. Through the investigation of patients in the hospital, the feasibility of the model was verified, and the characteristics of pressure ulcer in hospitalized patients in China were obtained. Through the interview with relevant medical experts, the model was improved [11], and the patients in the model were classified and analyzed to determine the occurrence of pressure ulcer. According to the relevant reasons, the paper puts forward targeted prevention suggestions, which have a strong reference role for the future research [12]. However, some of these studies have no universal value due to the limitation of samples and the lack of research methods, which can only provide some reference opinions.

This paper sorts out and classifies the data obtained by the literature research method, which provides a theoretical basis for the conception and research analysis of this paper, and makes a detailed analysis on the Internet of Things. It opens up a new theoretical perspective for deepening scholars' understanding of Internet of Things medical treatment; according to the research problems, the questionnaire is designed to combine the theoretical research and empirical research to analyze the causes of pressure ulcer and the prevention of pressure ulcer, which can enrich the relevant scholars' research on pressure ulcer and has certain research significance.

\section{Internet of Things for the Prevention of Pressure Ulcers}

2.1. Internet of Things Healthcare. Through the Internet of Things, medical personnel can interact with each other through the Internet of Things and medical devices [13]. Through the comprehensive application of barcode identification, wireless sensor network, and other Internet of Things technologies, it realizes the identification, regional positioning, tracking and management of special groups, medical devices, medical waste, and drugs in the hospital. The aspects that Internet of Things can play a role in the management process of medical and health fields include the following: diagnosis and treatment sign input, patient identity management barcode, mobile doctor's order, inspection specimen movement management, drug mobile management, baby antitheft, medical record management, movement number, data transfer and preservation, and nursing process management [14-16]. And, in the field of medical care, it can monitor the various conditions of the human body and transmit the data to various communication terminals. Its main application is in the monitoring of the human body and the measurement of physiological parameters. The monitoring objects include not only patients but also healthy people [17].

In the medical Internet of Things, due to the huge amount of collected data, on the one hand, it realizes the effective collection and integration of medical information, which makes health promotion possible. On the other hand, it is unavoidable to integrate and share massive multisource heterogeneous data in the network. These information include data, images, audio, and text data [18]. After intelligent processing of the collected information, real-time health file information resources are obtained, and according to the corresponding real-time monitoring data, personal health promotion reports are made, and various forms of tracking are carried out [19]. These make the information in intelligent health management from collection and processing to application, showing significant multisource heterogeneous characteristics. The transmission of information is data centric. Due to the large number of sensor nodes and random distribution, the data obtained by monitoring the same event between adjacent sensor nodes are similar. However, the storage space and energy of sensor nodes are limited, and the characteristics of dense distribution of largescale sensor nodes lead to most of the collected data become redundant. It will shorten the lifetime of the whole network and reduce the efficiency of information integration. At the same time, the network involves a large number of spatial distribution of health data, medical information, physical examination data, health measurement and evaluation, equipment operation and parameters, and other decentralized system space-time data [20,21].

Internet of Things technology can help hospitals to achieve intelligent medical care for people and intelligent management of things, which has great potential in the medical field. Moreover, it supports the digital collection, processing, storage, distribution, transmission and management of personnel 
management information, equipment information, medical information, and drug information within the hospital and can meet the needs of medical equipment and supplies, medical and health-related information, intelligent supervision of public health safety, etc. It can realize the visualization of material management, the digitization of medical information [22], the digitization of the medical process, the scientificization of the treatment process, and the humanization of service communication, so as to solve various problems such as the overall low level of medical service, the weak cultural support of medical cooperation, and the annual production hidden trouble of medical safety [23].

As shown in Figure 1, there is a huge amount of data information in the medical Internet of Things. With the continuous increase of data collected from medical sensors and new measurement equipment, how to obtain effective data from the sign sensor network and environmentalsensing equipment is a very critical problem [24]. Therefore, it is necessary to use effective data fusion technology to process the relevant data [25]. In order to reduce the data delay in the network, a fusion path method with minimum delay is proposed:

$$
T_{n}(i)=\delta \frac{r_{i}}{w_{i}} \cdots(i \in N)
$$

The specific algorithm is as follows:

$$
f=\left[-\frac{1}{2} \sum_{c=1}^{i} \sum_{d=1}^{i}\left(a_{c}^{*}-a_{c}\right)\left(a_{c}-a_{c}^{*}\right) Q\left(x_{c}, x_{d}\right)-\vartheta \sum_{c=1}^{i}\left(a_{c}^{*}+a_{c}\right)+\sum_{c=1}^{i} b_{c}\left(a_{c}^{*}+a_{d}\right)\right] \text {. }
$$

Among them,

$$
\sum_{c=1}^{i} a_{c}^{*}=\sum_{c=1}^{i} a c a_{c}^{*}, \quad a_{c} \in[0, D](c=1,2,3, \ldots, m),
$$

where $D$ is a normal number, which is called the penalty factor. If the value of $D$ is large, it means that the penalty for fitting deviation is large. At this time, the regression function can be expressed as

$$
V=\sum_{c=1}^{i}\left(a_{c}^{*}-a_{c}\right) Q\left(x_{c}, x\right)+s
$$

when $a_{c} \in(0, D)$,

$$
s=d_{c}-\sum_{c=1}^{i}\left(a_{c}^{*}-a_{c}\right) b\left(x_{c}, x\right)+\vartheta
$$

when $a_{c}^{*} \in(0, D)$

$$
s=d_{c}-\sum_{c=1}^{i}\left(a_{c}^{*}-a_{c}\right) b\left(x_{c}, x\right)-\vartheta .
$$

2.2. Pressure Sore. Pressure ulcer refers to the soft tissue damage caused by continuous hypoxia, ischemia, and malnutrition caused by long-term compression and blood circulation disorder in local tissues, such as ulceration and necrosis [26].

The risk factors of intraoperative pressure sores are as follows:

(1) The operation time is long: long operation time is one of the main risk factors leading to pressure ulcers. Some studies have shown that when the local tissue pressure time is greater than 2.5 hours, the incidence of pressure ulcers will be significantly increased. When the operation time exceeds 3 hours, the incidence of pressure ulcers is more than $8.5 \%$, and the operation time of extracorporeal circulation surgery generally exceeds 3 hours, so intraoperative pressure ulcers are prone to occur [27].

(2) Intraoperative hypothermia: hypothermia during cardiopulmonary bypass can directly damage the immune function of the body, reduce the blood oxygen supply of the tissue, resulting in ischemia, hypoxic injury of the skin, and subcutaneous tissue and muscle, and produce reversible or irreversible damage to the cells and microvessels. The incidence rate of pressure ulcer is also an important reason.

(3) The patient's reaction under anesthesia is relatively slow, and the body cannot produce protective response.

(4) The use of high-dose vasoactive drugs can cause peripheral vasoconstriction, lead to tissue ischemia and hypoxia, and promote the occurrence of pressure ulcers.

(5) The influence of lying position: the position of cardiopulmonary bypass surgery is the chest supine position. On the basis of general supine position, a position pad is placed on the shoulder blade, which can fully expose the operation field and facilitate the operation. But this kind of forced operation position will reduce the area of the patient's body on the mattress and increase the local pressure of the focus point, so it will increase the probability of intraoperative pressure sores.

(6) Patient factors: the patient's body shape, age, past medical history, and nutritional status will affect the occurrence of pressure ulcer. When the patient's body is too fat or too thin, it will increase the incidence of pressure sores [28]. Because the patient's weight is directly proportional to the degree of local tissue pressure, when lying in bed, the greater the weight, the greater the pressure on the local skin, the 


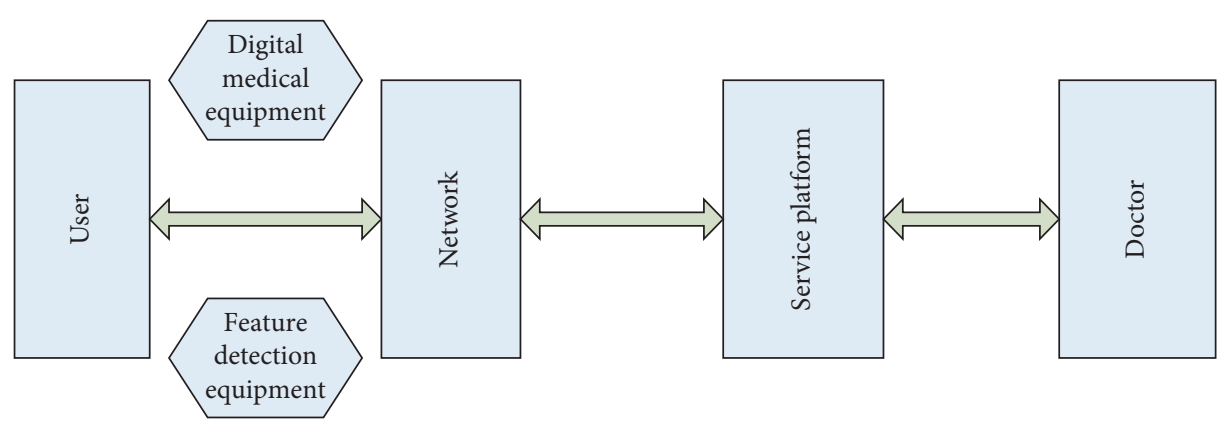

FIgURE 1: Internet of Things healthcare.

more prone to pressure ulcers. When the patient's body shape is too thin, because of skin relaxation, subcutaneous lack of fat tissue on the pressure buffer, and lying in bed, subcutaneous tissue blood vessels are squeezed, and tissue blood circulation perfusion is poor so that the skin pressure tolerance is decreased, leading to the occurrence of pressure ulcers.

In the process of pressure ulcer healing, the most important thing is to prevent infection, which directly affects the degree and time of wound healing. Infection means that bacteria, viruses, fungi, and other microorganisms invade the human body or propagate in a large number in the body, showing local swelling, heat and pain, inflammatory exudate or pus, or accompanied by chills, high fever, and other systemic inflammatory reactions. The wound is red and swollen, and the patient has obvious pain [29]. A large number of leukocytes are found in the local exudate to phagocytize and remove foreign bodies. The exudate fibrin gradually solidified to form a protective barrier, which could prevent the invasion of bacterial toxins. The self-protection function of the body can control the infection to a certain extent. In clinic, anti-infective drugs can be given intravenously, and local dressing change can be used to relieve the symptoms of high fever and pain.

As long as we pay attention to and take corresponding protective measures, most pressure sores can be prevented. The measures commonly used to prevent pressure sore in clinic include turning over regularly, keeping the bed clean and dry, increasing the intake of nutrition for patients, using air cushion bed, and sticking pressure sore patch (including silver antibacterial dressing, hydrocolloid dressing, alginate dressing, etc.) at the place prone to pressure ulcer [30]. There are special preventive measures for pressure ulcer during operation. Due to the difference of various operations (operation time, operation method, operation position, etc.), there is no prospective study to prove which intervention measures can effectively avoid intraoperative skin damage and prevent pressure ulcer.

Pressure ulcer is an important standard to evaluate the quality of nursing in the clinical work. It is very important to prevent the occurrence of pressure ulcer. As long as we pay more attention to it and carry out effective prevention, the probability of pressure ulcer can be greatly reduced. Therefore, it is particularly important to take feasible and effective preventive measures [31].
When the patient's body is too fat or too thin, it will increase the risk of pressure sores. Because the patient's weight is directly proportional to the degree of local tissue pressure, when the patient's body is too fat, the greater the weight, the greater the pressure on the local skin, and the more prone to pressure ulcers. When the patient's body shape is too thin, because of skin relaxation, subcutaneous lack of fat tissue on the pressure buffer, and lying in bed, subcutaneous tissue blood vessels are squeezed, and tissue blood circulation perfusion is poor so that the skin pressure tolerance is decreased, leading to the occurrence of pressure ulcers. The older the patient is, the more likely it is to develop pressure ulcer. Due to the lower sensitivity and vitality of motor and nerve, slow sensory function, decline of protective reflex, poor peripheral circulation, dry and loose skin, obvious atrophy, and thinning of the subcutaneous tissue, elderly patients are more likely to suffer from ischemia and hypoxia after local skin and subcutaneous tissue compression, leading to pressure ulcers [32].

Intraoperative application of glucocorticoids and vasoactive drugs will affect the occurrence of pressure ulcers; the use of active drugs will make blood vessels dilate, blood flow slow, and tissue and organs lose normal blood circulation, resulting in ischemia and hypoxia of surrounding tissues, which is easy to lead to pressure ulcers; the use of anesthetic drugs during operation will make skeletal muscle relaxation, loss of consciousness, weakening of muscle tension, and temporary loss of protective response to physical discomfort, leading to the occurrence of pressure ulcers [33].

Due to the lack of knowledge of pressure ulcer prevention and treatment in elderly patients with chronic diseases, the wound surface was deep when they were admitted to hospital, accompanied with different degrees of infection, obvious pain, and long treatment time. In this case, the local dressing change is suitable. The clinical inpatients agreed with the local dressing change prescription.

2.3. Foam Dressing. Foam dressing can improve the number of leukocytes in patients with deep pressure sores, which is consistent with other studies. The hydrocolloid components in foam dressing can promote autolysis, limit inflammation, induce hypoxic tension, and stimulate the release of macrophages and interleukin. Research shows that the use of foam dressing in the incision of tracheotomy in stroke patients can change dressing frequency, reduce frequency of wound dressing, and effectively reduce the infection rate. 
Foam dressing has antibacterial effects. It can effectively kill Staphylococcus aureus and Escherichia coli, improve the level of inflammatory cells, promote the mediating response of wound inflammatory response, and promote healing. This study shows that there is no difference in the effect of dressing change on white blood cells of cancer patients, which may be due to the short intervention time of the two methods, and the wound surface of deep pressure ulcer is not fully healed within 4 weeks, and the bacteriostatic effect is not effectively expressed [34].

In the process of dressing change, the necrotic tissue needs to be removed timely after the wound infection is controlled; otherwise, the growth of the new granulation tissue will be affected. Wound healing is the stage of granulation proliferation and epithelial creeping [35]. At this time, local blood circulation is accelerated, tissue metabolism is vigorous, nutrient transport is accelerated, cytokine activity is enhanced, and a large number of fibroblasts proliferate, which makes the capillary network form rapidly and accelerate the wound healing.

Different support tools can be selected according to different parts of pressure ulcer. Sacrococcygeal pressure sore can guide patients to make hollow thick sponge pad under the body, knee pressure sore with soft pillow between the two legs, ankle pressure sore can be made of rubber gloves to pad under the foot, and so on. These measures can effectively prevent the wound from deteriorating. Medical staff should make reasonable plan according to the stage of pressure ulcer, wound condition, and objective data of nursing evaluation.

In addition to taking auxiliary measures, pressure, an important factor in the formation of pressure ulcers, should be paid enough attention. The mechanism of pressure ulcer is that when the external pressure is greater than the capillary pressure, the inner diameter of capillary and lymphatic lumen is reduced, the blood flow is blocked, oxygen-carrying red blood cells pass through the barrier, nutrients cannot be supplied, and the accumulation of metabolites delays the wound healing. The most effective measure to relieve local pressure is to turn over frequently. Nursing staff should attach great importance to the factors of potential pressure ulcers. Patients should use high-standard reactive (constant low pressure) or active (pressure alternating) support surface whether in bed or in a wheelchair for a long time, so as to prevent the progression and deterioration of the pressure ulcer wound [36]. The choice of the supporting surface is different according to the position, stage, quantity, and potential risk of pressure ulcer. The pressure sore wound should be suspended and cannot continue to be stressed. Patients and their families should be instructed to place the patient's position reasonably and turn over frequently. The frequency should be adjusted according to the patient's feeling and the characteristics of the support surface selected [37].

\section{Internet of Things Medical Foam Dressing to Prevent the Pressure Ulcer Test}

3.1. Purpose of the Experiment. Based on the theoretical results of preventive medicine and drawing lessons from the research results of big data integration theory at home and abroad, this paper carries out a deep analysis of the causes of pressure ulcers and the differences between different prevention methods for patients by using the methods of literature review, comparative study, mathematical statistics, and logical analysis.

3.2. Experimental Evaluation Criteria. Entropy method is a relatively objective evaluation index weight assignment method, which can effectively avoid the subjectivity of artificial scoring and has high accuracy. But, at the same time, this study also realized that the entropy method cannot directly reflect the knowledge, opinions, and experience judgment of experts and scholars, and the weight results may be contrary to the actual situation. Therefore, this paper uses AHP and entropy method to determine the weight coefficient of the regional higher education evaluation index.

3.3. Data Sources. The data in this paper are mainly from the First People's Hospital of a city. We conducted a survey on patients of different ages in the hospital and collected relevant data through case and doctor interviews.

3.4. Pressure Ulcer Prevention Model Method. The patients were parameterized to prevent pressure ulcers and then transmitted to the data model. The data module receives the needs of users, finds the appropriate resources through resource evaluation, performs tasks, and completes user requests.

The data module receives the request from the user and transmits it to the general server in the local area network [38]. Monitoring devices track the resource utilization and availability of sensors, applications, and services, generate statistical logs, and transmit data to the server. The server receives the user's request from the mobile terminal, analyzes the user's request information according to the monitored resource information, divides the service into several tasks, and processes the calculation. According to the evaluation results, each task selects the best matching resource from the resource pool, schedules and allocates resources, and provides the optimal prevention method for patients [39].

Considering the resources with the same service function, the attributes of the resources are set according to the user preferences, which have certain scalability. The calculation formula is as follows: 


$$
U_{a}=p * b \frac{j}{i} * c
$$

In order to calculate the weight of patient attributes and ensure the objectivity of evaluation results, we use the entropy weight method to determine the entropy value and entropy weight of each resource attribute:

$$
\begin{aligned}
& t=\frac{1}{\ln x} \sum_{n=1}^{1} f_{n m} * \ln f_{n m} \\
& r=\frac{1-t}{y-\sum_{m=1}^{m} t} .
\end{aligned}
$$

Among them,

$$
\begin{aligned}
f_{m n} & =\frac{z_{n m}}{\sum_{n=1}^{x} z_{n m}}, \\
\sum_{m=1}^{x} w_{m} & =1 .
\end{aligned}
$$

Based on the attribute value of the user request resource, each resource is regarded as a point in the multidimensional space, and the Euclidean distance is used to measure the proximity between the resource and user demand due to the user's preference for a certain attribute or each attribute of the resource has different influence on the measurement result. Therefore, the objective weight of each user is set to

$$
\begin{aligned}
& d=\sqrt{\sum_{m=1}^{x} w_{n} *\left(r_{n m}-u q_{m}\right)^{2}} \\
& d_{n}=\frac{1}{1+d\left(r_{n}, u q\right)} .
\end{aligned}
$$

The similarity between the available resources and the resources requested by the user is obtained, and the threshold value is set in the range of $[0,1]$. Thus, the matching value $Q$ can be obtained:

$$
Q_{\delta}=\left\{r_{n}\left|d \cos \left(r_{n}, u q\right)\right| \geq \delta\right\} .
$$

The similarity between the resources in the matching resource set $Q$ and the resources requested by users is calculated:

$$
\cos m(r, u q)=\alpha * \cos (r, u q)+(1-\alpha) * \frac{1}{m} \sum_{n=1}^{m} \delta_{i j},
$$

where $a$ is the weight and the range is between $[0,1]$. The simulation parameters are calculated as follows:

$$
F=\frac{\sum\left(q_{r p, r p n}-q_{r p m}\right)\left(q_{u q, u q n}-q_{u q}\right)}{\sum_{n=1}^{n}\left(q_{r}-q_{u q}\right)^{2} \sqrt{\sum\left(q_{u q, u q m}-q_{u q}\right)^{2}}} .
$$

\section{Experimental Analysis of Foam Dressing to Prevent Pressure Sore}

4.1. Distribution of Patients with Pressure Ulcer. We made statistics on 100 male patients with pressure ulcers and classified them according to their gender and age. The specific statistics are shown in Table 1, as shown in Figures 2 and 3 .

From the chart, we can see that there are obvious differences between pressure ulcer patients. Among the investigated population, only $18 \%$ of the people under 40 years of age have pressure ulcer, and those over 40 years have pressure ulcer much higher than those under the age of 40 . In particular, the proportion of pressure ulcer in people over 60 years old accounts for more than $50 \%$, which shows that the elderly group is affected by pressure ulcer and the impact is the most serious.

4.2. Causes of Pressure Ulcer. For the reason of pressure ulcer, there is no public opinion at present; we, through the investigation of patients, determine the body parameters of patients with pressure ulcers and the abnormal place of ordinary people, in order to find the reasons for pressure ulcers. The details are shown in Table 2 and Figures 4 and 5.

From the chart, we can see that, before and after the emergence of pressure ulcers, the patient's body temperature, operation time, and operation cardiopulmonary bypass time have varying degrees of change. Among them, patients with pressure ulcers, their nasopharyngeal temperature and lower body temperature are different from the normal temperature; a low-temperature environment may lead to the emergence of pressure ulcers; in addition, after the operation time is more than 3 hours, the frequency of pressure ulcers is much higher than that of less than 3 hours of operation time, and the external circulation time is also the same. We can infer the relationship between bedsores and the patient's body temperature and operation time.

4.3. Preventive Effect of Foam Dressing on Pressure Sore. We selected 100 men and 100 women in the most prone to pressure sores (over 60 years old). A control experiment was carried out. 100 of them used traditional treatment techniques instead of foam dressing. 100 patients were treated with foam dressing to control pressure ulcers. Specific data are shown in Table 3 and Figures 6 and 7.

According to the chart, we can see that, in the experimental group and the control group, there were 15 pressure sores in 100 cases of the experimental group, while only 7 of the control group had pressure sores. It is obvious that foam dressing can play a key role in the prevention of pressure sore.

4.4. Medical Role of Internet of Things. Through the above experiments, we can test that foam dressing can play a role in the prevention of pressure sore. In order to improve the relative preventive effect, we compare the manual foam 
TABLE 1: Distribution of patients.

\begin{tabular}{lcccc}
\hline & $0-20$ & $21-40$ & $41-60$ & \\
\hline Male & 5 & 12 & 24 & 59 \\
Female & 3 & 16 & 31 & 50 \\
Mild pressure sore & 7 & 21 & 18 & 33 \\
Severe pressure sore & 1 & 7 & 37 & 76 \\
\hline
\end{tabular}

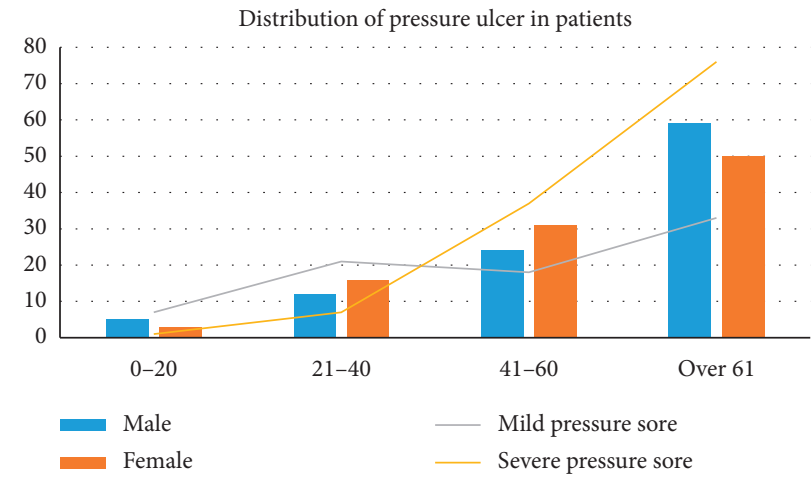

FIGURE 2: Distribution of pressure ulcer in patients.

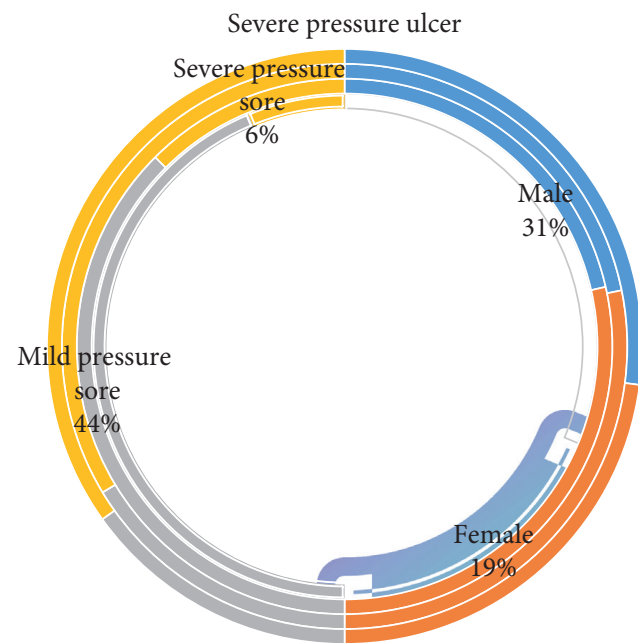

FiguRE 3: Severe pressure ulcer.

TABLE 2: Distribution of patients.

\begin{tabular}{lcccc}
\hline & Nasopharynx temperature & Lower body temperature & Operation time & Cardiopulmonary bypass time \\
\hline Male patients & 34.21 & 35.32 & 4.25 & 3.59 \\
Female patients & 35.33 & 36.42 & 4.21 & 3.69 \\
Normal men & 37.35 & 37.33 & 3.58 & 2.34 \\
Ordinary women & 37.15 & 37.52 & 3.32 & 2.45 \\
\hline
\end{tabular}

dressing with the Internet of Things medical equipment. The specific data are shown in Table 4 and Figure 8.

From the chart, we can see that foam dressing can reduce the occurrence of pressure sores and reduce the probability of pressure ulcers by about $30 \%$. Compared with the unused pressure sore, the use of IoT can increase the efficiency of foam dressings and reduce the probability of pressure sore by about $7 \%$. This shows that the auxiliary foam dressing of medical devices in the logistics network can play an important role in preventing pressure sore. 


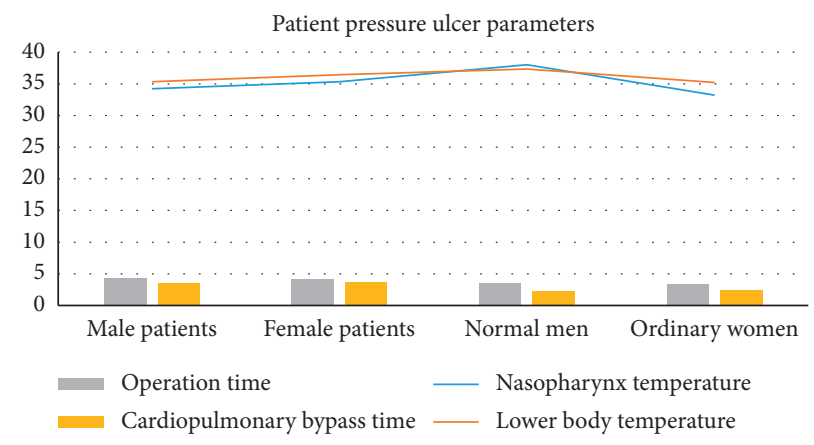

Figure 4: Patient pressure ulcer parameters.

Changes of pressure ulcer index

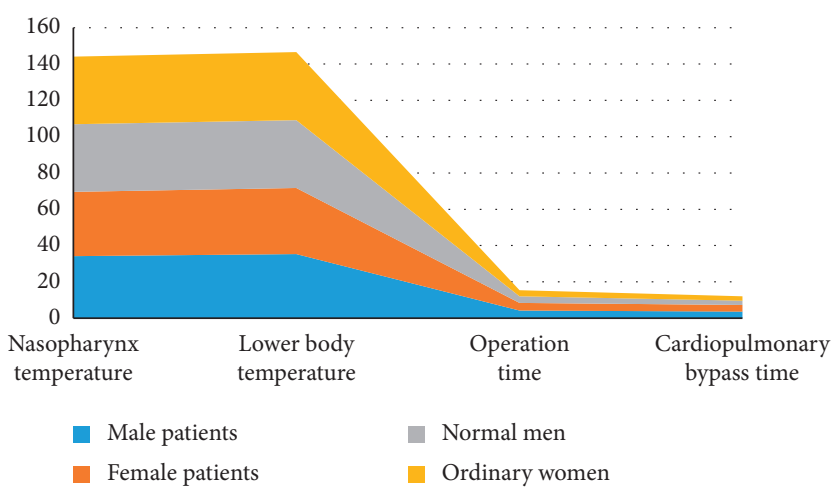

FIgure 5: Changes of the pressure ulcer index.

TABle 3: Distribution of patients.

\begin{tabular}{lccccc}
\hline & $\begin{array}{c}\text { Number of people over } \\
\text { temperature }\end{array}$ & $\begin{array}{c}\text { Long operation } \\
\text { time }\end{array}$ & $\begin{array}{c}\text { Number of } \\
\text { inflamed patients }\end{array}$ & Turnover care & Number of pressure ulcers \\
\hline Experience group & 37 & 43 & 32 & 50 & 15 \\
Control group & 39 & 52 & 21 & 50 & 7 \\
\hline
\end{tabular}

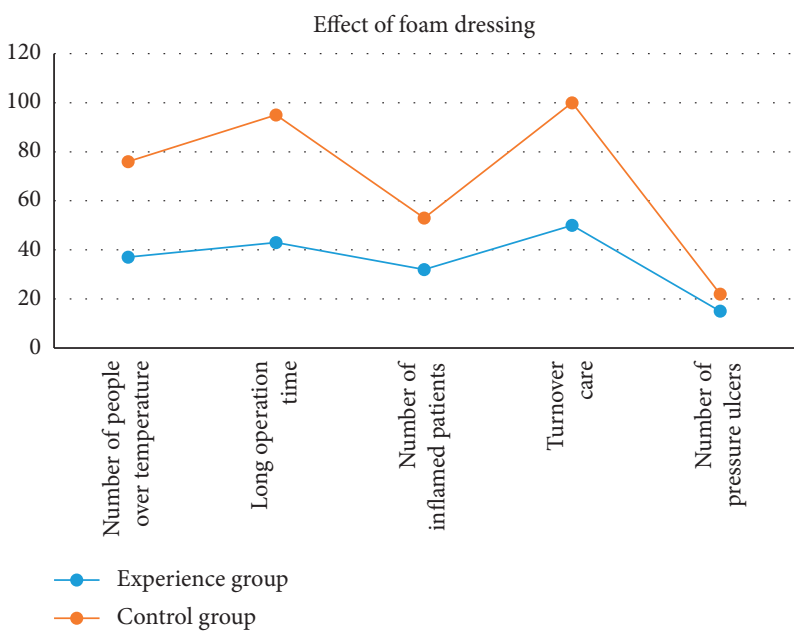

FIgURE 6: Preventive effect of foam dressing on pressure sore. 


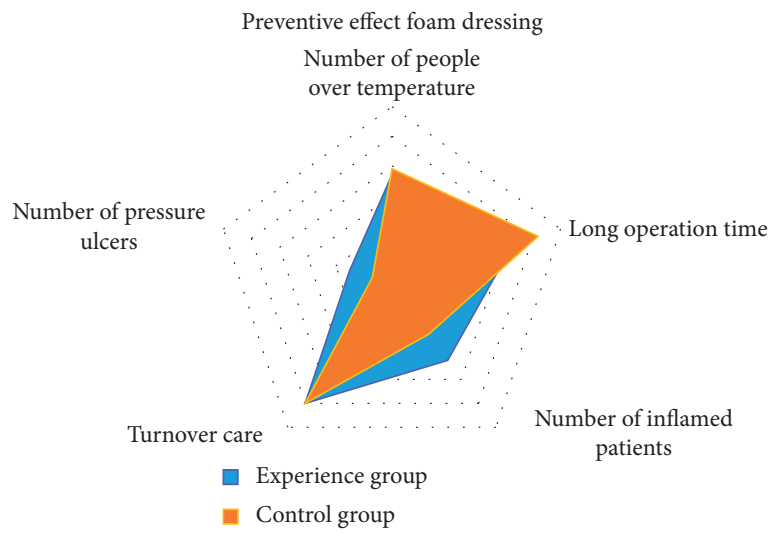

Figure 7: Preventive effect of foam dressing.

TABLE 4: Distribution of patients.

\begin{tabular}{|c|c|c|c|c|}
\hline & Primary pressure ulcer & Secondary pressure ulcer & Stage-III pressure ulcer & Stage-IV pressure ulcer \\
\hline Labor group & 6 & 4 & 5 & 3 \\
\hline Internet of Things group & 3 & 1 & 2 & 1 \\
\hline
\end{tabular}

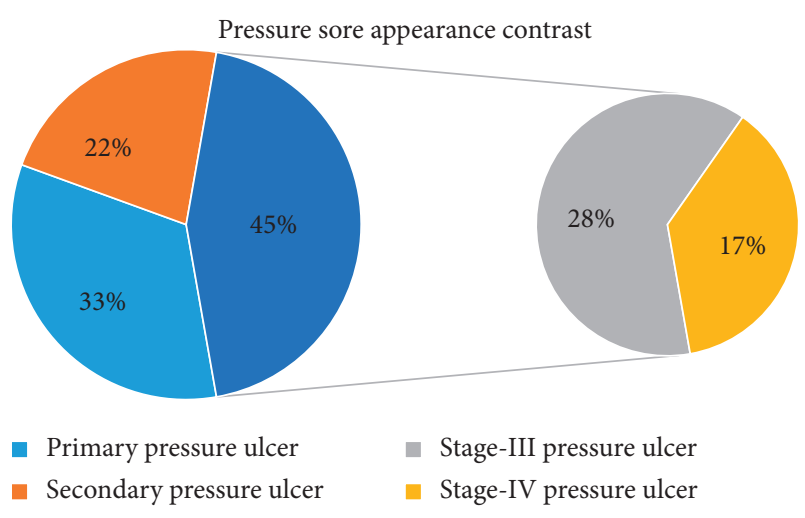

FIgURE 8: Pressure sore appearance contrast.

\section{Conclusions}

Foam dressings are mainly made of polyvinyl alcohol foam and polyurethane. They are soft, elastic, and have strong absorbency. The main component of foam dressing is soft silicone adhesive, which is made of water, oil, and silicon. Skin contact without irritation, soft, with a certain thickness, can reduce the discomfort and pain caused by local compression. It can buffer the local pressure. The new dressing has the function of air permeability and waterproof, which can reduce the symptoms of skin immersion and local hyperemia and play a good barrier role. The high absorptive capacity and decompression ability of clinical foam dressings have achieved good results in clinical application.

The occurrence of pressure ulcers aggravates the physiological and psychological pain of patients, causes huge economic losses, and increases the workload of nurses, which leads to some medical disputes and takes up a large number of public health resources. The prevention of pressure ulcer is one of the important problems to be solved urgently in the clinical nursing work and is a very important reference index to evaluate the quality of nursing.

Deep pressure ulcer has become one of the serious complications that perplex patients and medical staff. It not only affects the recovery of primary disease but also brings a series of more difficult serious complications. The pain torture makes the patients miserable, and the infection secondary to the wound seriously affects the health and quality of life of patients and even threatens their lives. There are many risk factors and causes of pressure ulcer, and the mechanism is relatively complex. The treatment and nursing of pressure ulcer need multidisciplinary comprehensive treatment. The current clinical treatment methods for wound dressing have a wide variety of drugs and their curative effects are also uneven as the main clinical treatment, a wide variety of drugs, curative effect is also uneven. How to choose an economical, appropriate, safe, and effective method of dressing change for patients is worthy of further study in the clinical nursing work.

\section{Data Availability}

The data underlying the results presented in the study are available within this paper.

\section{Conflicts of Interest}

The authors declare that they have no conflicts of interest.

\section{References}

[1] C. Yang and Z. Lv, "Gender based face aging with cycleconsistent adversarial networks," Image and Vision Computing, vol. 100, Article ID 103945, 2020.

[2] L. Lin, "Application of foam dressing in prevention of facial pressure sores in patients with noninvasive mechanical 
ventilation," World's Latest Medical Information Digest, vol. 1, no. 66, pp. 130-131, 2015.

[3] K. G. Srinivasa, B. J. Sowmya, A. Shikhar, R. Utkarsha, and A. Singh, "Data analytics assisted internet of things towards building intelligent healthcare monitoring systems: iot for healthcare," Journal of Organizational and End User Computing, vol. 30, no. 4, pp. 83-103, 2018.

[4] Y. Sun, H. Song, A. J. Jara, and R. Bie, "Internet of things and big data analytics for smart and connected communities," IEEE Access, vol. 4, pp. 766-773, 2016.

[5] M. Elhoseny, G.-B. Bian, S. K. Lakshmanaprabu, K. Shankar, A. K. Singh, and W. Wu, "Effective features to classify ovarian cancer data in internet of medical things," Computer Networks, vol. 159, pp. 147-156, 2019.

[6] X. Ma, C. Jiang, and Y. Yang, "Application of non adhesive ankle foam dressing to prevent nasal facial pressure sores in patients undergoing noninvasive mechanical ventilation," Chinese Journal of Pulmonary Diseases (Electronic Version), vol. 8, no. 4, pp. 106-107, 2015.

[7] W. Xiangfeng, "The effect of Wu Xiangfeng foam dressing in preventing pressure ulcers caused by respirator mask," Integrated Traditional Chinese and Western Medicine Nursing (English, Chinese), vol. 1, no. 3, pp. 87-89, 2015.

[8] R. Chen, Y. Wang, Y. Qi et al., "Effect of foam dressing on prevention of nasal facial pressure ulcers in patients with ICU noninvasive mechanical ventilation," Journal of Practical Medicine, vol. 32, no. 7, pp. 1180-1182, 2016.

[9] L. Shi, H. Zhang, J. Zhang et al., "Using soft silicone foam dressing to prevent pressure ulcer in critically ill patients after trauma. randomized controlled clinical trial," China Health Nutrition, vol. 26, no. 27, pp. 50-51, 2016.

[10] M. Wang, "Application of foam dressing in prevention of pressure ulcer in critically ill patients in department of cardiology," Diet Health Care, vol. 6, no. 44, pp. 150-155, 2019.

[11] M. Abdel-Basset, M. Elhoseny, A. Gamal, and F. Smarandache, "A novel model for evaluation hospital medical care systems based on plithogenic sets," Artificial Intelligence in Medicine, vol. 100. , 2019 in press.

[12] C. Zhong, J. Liu, Q. Lai et al., "Application of foam dressing in preventing nasal pressure sores in patients with noninvasive ventilation assisted by department of respiration," China Contemporary Medicine, vol. 26, no. 5, pp. 212-214, 2019.

[13] W. Elsayed, M. Elhoseny, S. Sabbeh, and A. Riad, "Selfmaintenance model for wireless sensor networks," Computers \& Electrical Engineering, vol. 70, pp. 799-812, 2018.

[14] Y. Wang, "Application of foam dressing in prevention of pressure ulcers caused by respirator mask," Healthy People (Mid Version), vol. 11, no. 5, pp. 16-18, 2017.

[15] S. Fang, Y. Gao, Y. Liu et al., "Effect of variable temperature blanket combined with foam dressing on prevention of pressure ulcers during cardiopulmonary bypass," Qilu Nursing Journal, vol. 22, no. 8, pp. 94-95, 2016.

[16] M. Elhoseny and K. Shankar, "Reliable data transmission model for mobile ad hoc network using signcryption technique," IEEE Transactions on Reliability, vol. 69, no. 3, p. 1077. in press, 2020.

[17] L. Jing, "Application of soft silicone foam absorption dressings in prevention of scalp pressure ulcer in patients with severe craniocerebral injury," Nursing Care in English and Chinese Medicine, vol. 1, no. 4, pp. 65-67, 2015.

[18] C. Li, H. J. Yang, F. Sun, J. M. Cioffi, and L. Yang, "Adaptive overhearing in two-way multi-antenna relay channels," IEEE Signal Processing Letters, vol. 23, no. 1, pp. 117-120, 2016.
[19] X. Zhang, Y. Xiao, and J. Wu, "Kang whale foam dressing to prevent pressure sores in posterior spinal surgery," Nursing Practice and Research, vol. 13, no. 2, pp. 106-107, 2016.

[20] H. Chen and X. Zhao, "Effect of foam dressing on prevention of pressure ulcers at the needle handle of infant indwelling needle," Heilongjiang Medicine, vol. 1, no. 6, pp. 1242-1244, 2016.

[21] Z. Lv, "Security of internet of things edge devices," Software: Practice Experience, pp. 1-11, 2020.

[22] Y. Zhang, L. Sun, H. Song, and X. Cao, "Ubiquitous WSN for healthcare: recent advances and future prospects," IEEE Internet of Things Journal, vol. 1, no. 4, pp. 311-318, 2014.

[23] L. Li, L. Fang, M. X. Rong et al., "Clinical application of foam dressing in preventing high risk parts of pressure ulcers in surgical patients," Clinical Study of Chinese Medicine, vol. 1, no. 21, pp. 124-126, 2017.

[24] H.. Han, "Application of foam dressing in prevention of pressure ulcer in prone position of department of orthopedics," China Health Nutrition, vol. 25, no. 11, pp. 66-68, 2015.

[25] F. Xiao, "Multi-sensor data fusion based on the belief divergence measure of evidences and the belief entropy," Information Fusion, vol. 46, pp. 23-32, 2019.

[26] Y. Cao, "Application of foam dressing in prevention of high risk pressure sores in patients with cliquid," Journal of Liaoning Medical University, vol. 5, no. 1, pp. 166-168, 2015.

[27] Q. Zhang, H. Xiao, and G. Huang, "Application of foam dressing in treatment of pressure sores in primary hospitals," Nursing Practice and Research, vol. 23, no. 7, pp. 143-144, 2015.

[28] K. Wang Ping, "Whale foam dressing for prevention of pressure sore in long term bedridden patients with stroke," Modern Medical and Health, vol. 31, no. z2, pp. 75-76, 2015.

[29] Y. Zhao, L. Cai, and J. Shen, "Effect of observation window on foam dressing for prevention and treatment of pressure sore," Zhejiang Clinical Medicine, vol. 1, no. 20, pp. 171-172, 2018.

[30] L. Li, L. Fang, M. X. Rong et al., "Clinical application of foam dressing in preventing high risk parts of pressure ulcers in surgical patients," Clinical Study of Chinese Medicine, vol. 3, no. 17, pp. 153-154, 2017.

[31] L. Li, H. Huang, Y. Lin et al., "Clinical application of foam dressing to prevent inevitable pressure sores," China School of Medicine, vol. 29, no. 4, pp. 305-306, 2015.

[32] Q. Nie, "Effects of foam dressing in prevention of pressure sores in critical ill patients after orthopaedic surgery," Nursing Care of 2017 Patients with Chinese Medicine Combined with Western Medicine (Chinese and English), vol. 3, no. 4, pp. 36-37, 2017.

[33] C. Qiao, "Clinical application of foam dressing in the prevention of high risk pressure sores," Medicine Frontiers, vol. 1, no. 3, pp. 282-283, 2016.

[34] R. Fei, Y. Xu, G. Liu et al., "Comparative study of foam dressing and " $R$ " turning mat in the prevention of pressure ulcer in the sacrococcygeal region," Anhui Medicine, vol. 21, no. 9, pp. 1736-1738, 2017.

[35] S. Ouyang, L. Chen, C. Lu et al., "Study on the prevention of pressure sore by foam dressing combined with padded warm air blanket in low body weight infants undergoing cardiopulmonary bypass," Evidence Based Medicine, vol. 20, no. 1, pp. 61-64, 2020.

[36] T. Luo, "Nursing effect analysis of 30 degree turning over method combined with foam dressing to prevent pressure sore," Electronic Journal of Clinical Medicine Literature, vol. 6, no. 42, pp. 134-136, 2019. 
[37] G. Liu, X. Zhang, L. He et al., "The effect of decompression mattress and foam dressing on preventing bedsore after chest tumor operation," Nursing Care in Integrated Traditional Chinese and Western Medicine (English, Chinese), vol. 5, no. 11, pp. 70-71, 2019.

[38] X. Li, "Application of hydrocolloid dressing combined with foam dressing in the prevention of pressure ulcers in elderly patients," Famous Doctor, vol. 1, no. 12, p. 238, 2019.

[39] H. Lu, D. Yao, and Y. Wang, "Soft silicone foam dressing for prevention of pressure ulcers in critically ill patients after trauma," Dermatology and Venereal Diseases, vol. 41, no. 4, pp. 153-154, 2019. 